Supporting Information for

\title{
Photo-Driven Self-Healing of Arbitrary Non-Destructive Damage in Polyethylene-Based Nanocomposites
}

Peng Yu, Xiao-Shuai Guo, Rui-Ying Bao, Zheng-Ying Liu, Ming-Bo Yang, Wei Yang*

College of Polymer Science and Engineering, Sichuan University, State Key Laboratory of

Polymer Materials Engineering, Chengdu, 610065, Sichuan, People’s Republic of China.

*Corresponding author. Tel.: + 862885460130 ; fax: + 862885460130.

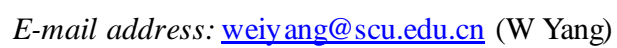




\section{SI-1}

FTIR spectrometer was used to characterize the sulfonation of MWCNT, as shown in Figure S1.

S-CNT synthesized with sulfanilic acid shows characteristic absorption peaks at 1571, 1499, 1432 $\mathrm{cm}^{-1}$ (aromatic $\mathrm{C}=\mathrm{C}$ stretching vibration), 698, 1031 and $1167 \mathrm{~cm}^{-1}\left(-\mathrm{SO}_{3}\right.$ stretching vibration), indicating that benzenesulfonic groups have been successfully grafted onto the surface of S-CNT. ${ }^{1}$ Thermogravimetric Analyzer was used to measure the concentration of functional groups on the surface of S-CNT. From Figure S1b, the weight of functional groups on the surface of S-CNT is about $6 \mathrm{wt} \%$ of S-CNT.
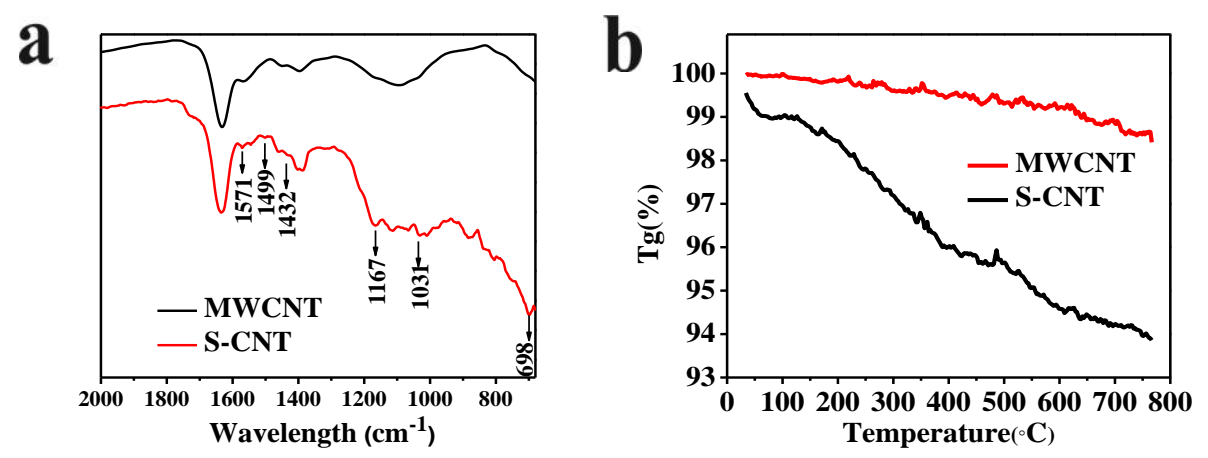

Figure S1. (a) FTIR spectra of MWCNT and S-CNT. (b) TG curve of MWCNT and S-CNT.

\section{SI-2}

Electron beams with different intensity were used to crosslink the HDPE matrix. The sample tends to turn yellow and degrade when the intensity of electron beam is strong, while the crosslinking density is too low when the intensity of electron beam is weak and as a result, the recovery of sample is not satisfying. So, the electron beam radiation of $100 \mathrm{kGy}$ was selected. For the evaluation of the crosslinking degree, sample HDPE, H/P/S-5/1, H/P/S-10/1, H/P/S-20/1, H/P/S-30/1 were washed with xylene through soxhlet extraction to remove the uncrosslinked parts. The crosslinking density of HDPE matrix in the samples was calculated and shown in Table S1. ${ }^{2}$ 
From the results in Table S1, it can be concluded that PU/S-CNT does not affect the crosslinking reactions of HDPE matrix significantly and the average crosslinking degree of HDPE matrix is roughly $41.6 \%$.

Table S1. Crosslinking degree of HDPE matrix in sample HDPE, H/P/S-5/1, H/P/S-10/1, H/P/S-20/1, H/P/S-30/1, evaluated by means of soxhlet extraction.

\begin{tabular}{cccccc}
\hline \multicolumn{1}{c}{ Samples } & HDPE & H/P/S-5/1 & H/P/S-10/1 & H/P/S-20/1 & H/P/S-30/1 \\
\hline Crosslinking degree & & & & & \\
of HDPE in sample & $41.7 \%$ & $42.8 \%$ & $40.7 \%$ & $40.7 \%$ & $41.9 \%$ \\
\hline
\end{tabular}

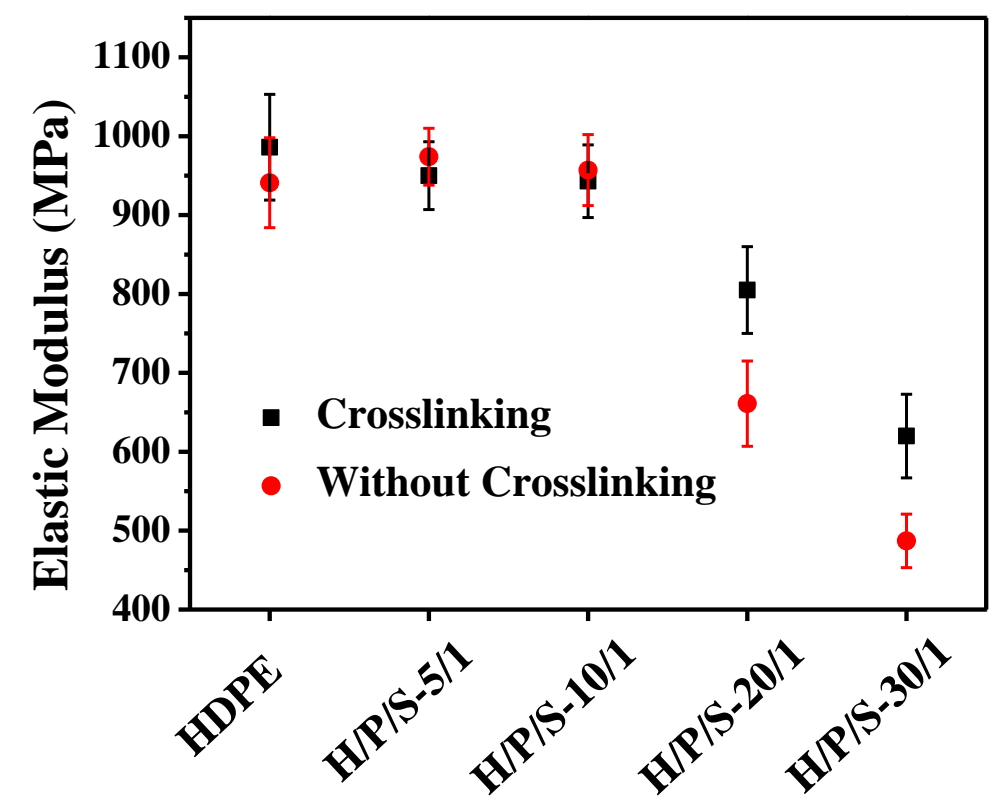

Figure S2. Elastic modulus of HDPE, H/P/S-5/1, H/P/S-10/1, H/P/S-20/1 and H/P/S-30/1. 


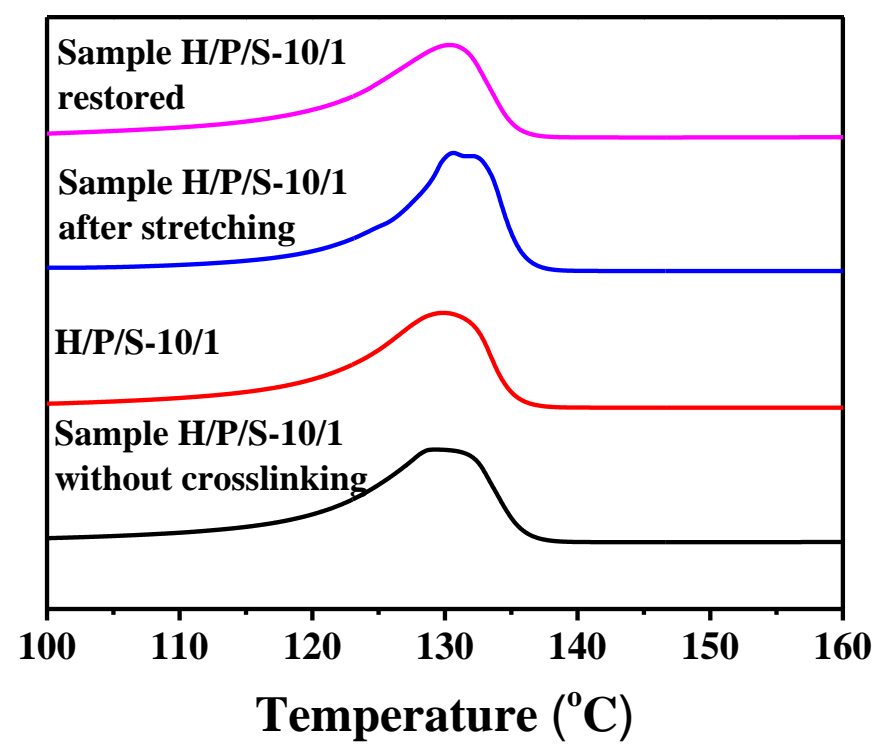

Figure S3. DSC curves of sample H/P/S-10/1 without crosslinking, H/P/S-10/1, sample H/P/S-10/1 after streching and sample H/P/S-10/1 restored.

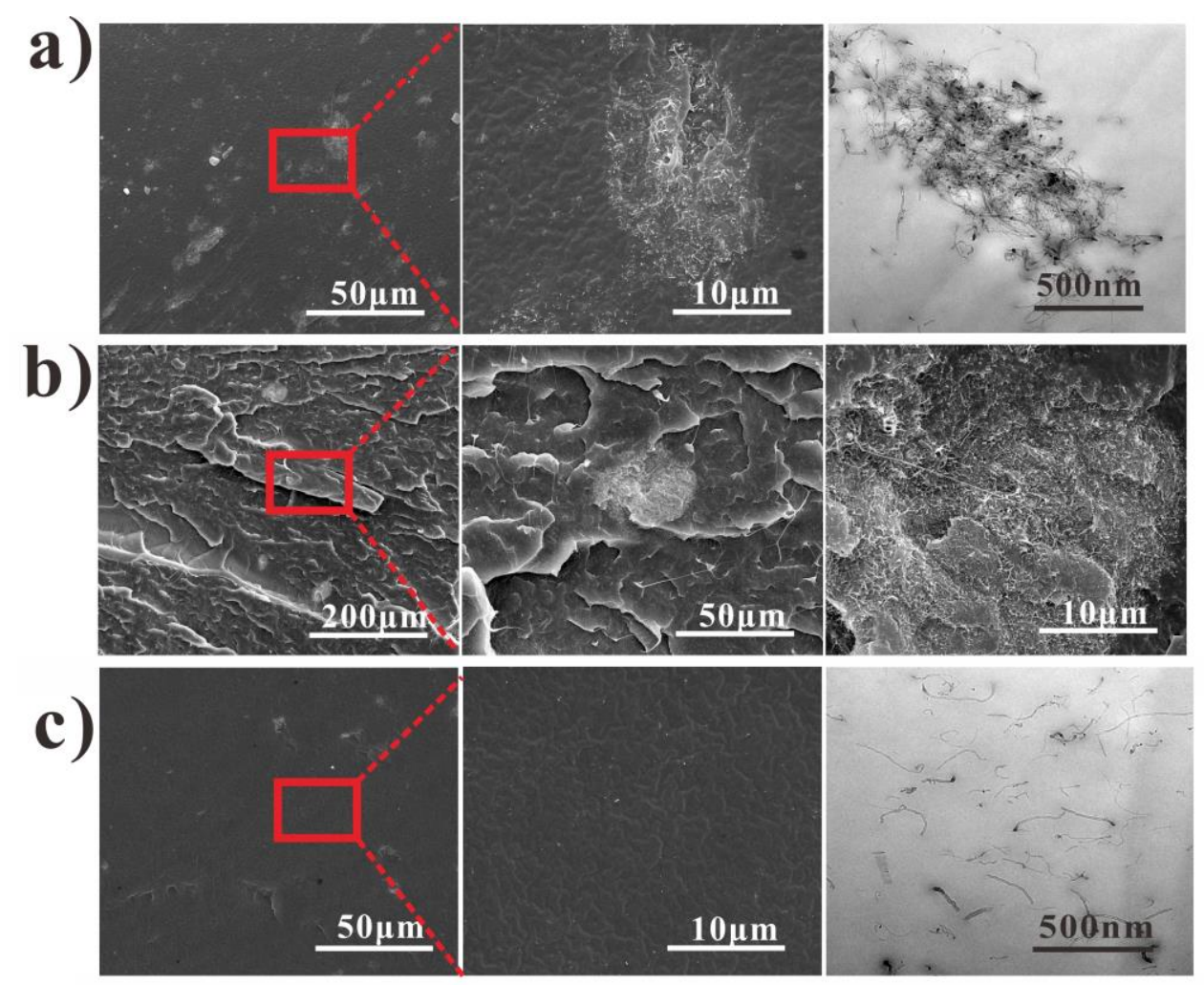

Figure S4. SEM and TEM images showing the (a) agglomeration of MWCNTs in PU, (b) the agglomeration of MWCNTs in H/C-0.1\% and (c) dispersion of S-CNT in TPU. 
a)

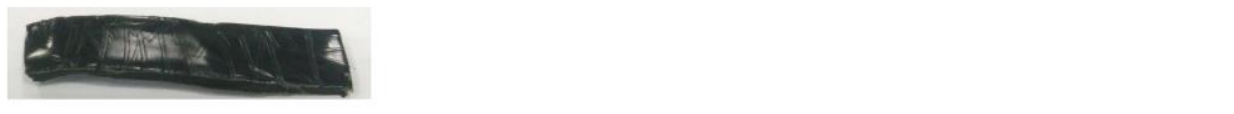

b)

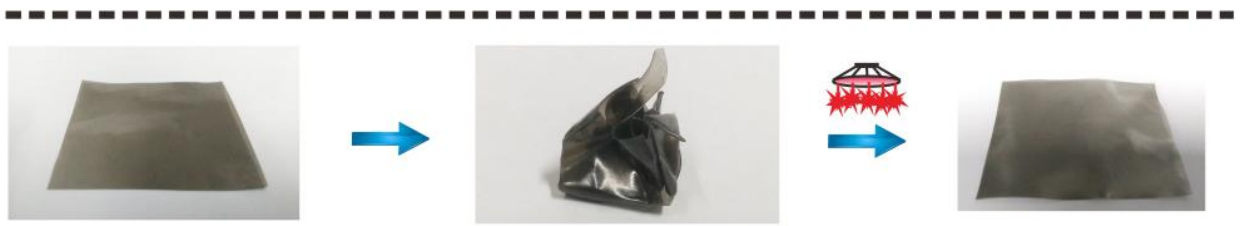

c)

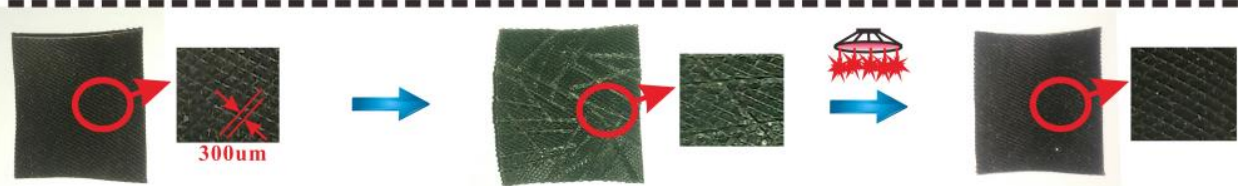

Figure S5. (a) The shape memory performance of W-H/P/S-10/1 after stretching, squeezing damage and twisted damage. (b), (c) The original, temporary and recovered shapes and dimensions of $\mathrm{H} / \mathrm{P} / \mathrm{S}-10 / 1$ composite in different shapes.

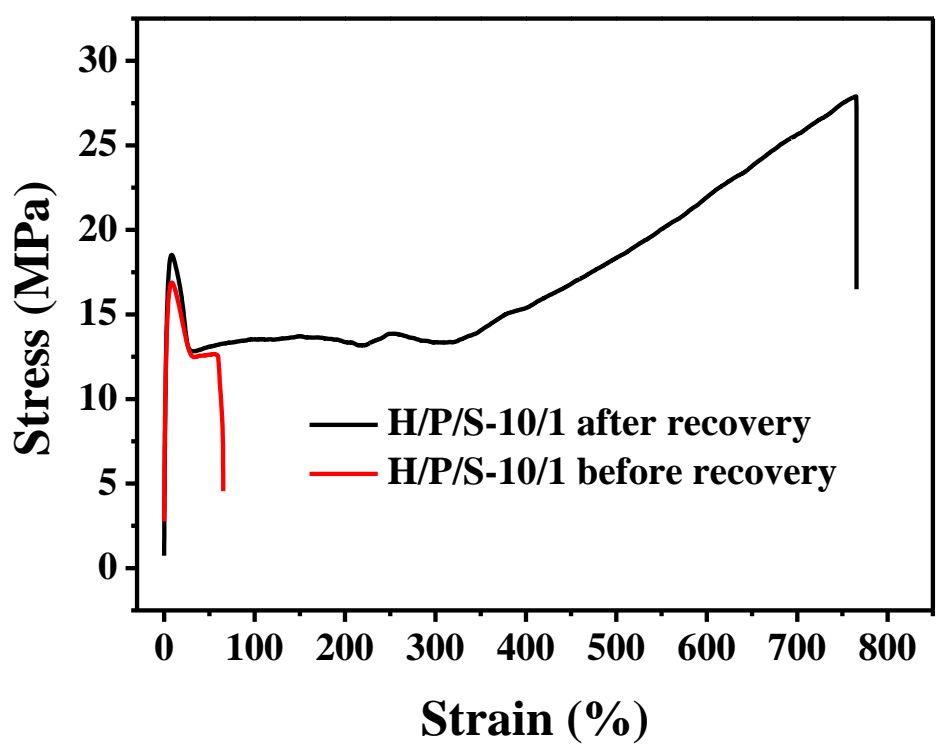

Figure S6. Stress-strain curve of $\mathrm{H} / \mathrm{P} / \mathrm{S}-10 / 1$ composite after extrusion destruction and the destructed composite after photo-driven recovery.

\section{References}

(1) Si, Y.; Samulski, E. T. Synthesis of Water Soluble Graphe. Nano Lett. 2008, 8, 1679-1682.

(2) de Sousa, F. D. B.; Scuracchio, C. H.; Hu, G. H.; Hoppe, S. Devulcanization of Waste Tire Rubber by Microwaves. Polym. Degrad. Stab. 2017, 138, 169-181. 